\title{
D-amino acid oxidase (DAO) rare genetic missense variant p.Pro103Leu and gastric cancer
}

\author{
YUAN ZONG $^{1}$, MASASHI TANAKA ${ }^{2,3}$, MASAAKI MURAMATSU $^{1}$ and TOMIO ARAI ${ }^{2}$ \\ ${ }^{1}$ Department of Molecular Epidemiology, Medical Research Institute, Tokyo Medical and \\ Dental University, Bunkyo-ku, Tokyo 113-8510; ${ }^{2}$ Department of Pathology, Tokyo Metropolitan \\ Geriatric Hospital and Institute of Gerontology, Itabashi-ku, Tokyo 173-0015, Japan
}

Received July 22, 2020; Accepted December 18, 2020

DOI: $10.3892 / \mathrm{mco} .2021 .2220$

\begin{abstract}
Gastric cancer is prevalent in the Asian population. Genetic predisposition to gastric cancer is not fully understood. Recent studies have demonstrated that D-amino acid oxidase (DAO), a multifunctional enzyme, protects the mucosa of gastrointestinal (GI) tracts by generating hydrogen sulfide $\left(\mathrm{H}_{2} \mathrm{~S}\right)$ in the stomach of rodents. The present study surveyed rare germline variants in the human DAO gene with regard to the incidence of gastric cancer. The consecutive autopsy cases registered in the JG-SNP database $(n=2,343$; mean age, 80 years) were employed and genotyped with Exome Bead-Chips. There were three non-synonymous rare variants, p.R22H, p.P103L and p.R283Q, of which the minor allele frequencies were $0.09,0.21$ and $0.02 \%$, respectively. Carriers of these variants were surveyed, the results of which revealed that 4 out of 10 patients with the p.P103L variant had gastric cancer (Fisher's exact test, $\mathrm{P}=0.018$ ). All 4 patients were men with drinking and smoking habits. Among the other 6 women, there was one incidence of small intestine cancer and one of colon cancer. Neither p.R22H nor p.R283Q carriers had GI cancer. DAO p.P103L is reported to be a modifier of amyotrophic lateral sclerosis (ALS) and may potentially be a hypomorphic allele. Thus, it is hypothesized that this rare variant might have affected protection against gastric mucosal damage through $\mathrm{H}_{2} \mathrm{~S}$ signaling in the mucosa, which leads to high prevalence of gastric cancer. The role of rare variant DAO p.P103L warrants further investigation in larger cohorts.
\end{abstract}

Correspondence to: Professor Masaaki Muramatsu, Department of Molecular Epidemiology, Medical Research Institute, Tokyo Medical and Dental University, 1-5-45 Yushima, Bunkyo-ku, Tokyo 113-8510, Japan

E-mail: muramatsu.epi@mri.tmd.ac.jp

Present address: ${ }^{3}$ Department of Neurology, Juntendo University Graduate School of Medicine, 2-1-1 Hongo, Bunkyo-ku, Tokyo 113-8421, Japan

Key words: D-amino oxidase (DAO) gene, rare variants, cancer predisposition, gastric cancer

\section{Introduction}

Gastric cancer is the fourth most common cancer worldwide, and $>50 \%$ of cases occur in Eastern Asia with nearly one million new cases and $>720,000$ deaths per year $(1,2)$. The risk factors of gastric cancer include race, sex, tobacco, alcohol, infection, and family history (3). The etiology of gastric cancer is still not fully known. While a battery of somatic mutations in driver genes have been identified, little is known about predisposing genes for gastric cancer. Aside from rare familial forms of gastric cancers, recent genetic analysis suggests that $\sim 10 \%$ of the sporadic cases may have rare variants with pathogenic implication $(1,4)$. The close digit of the incidence and mortality of gastric cancer reflects that only a limited fraction is detected at early stages for efficient intervention, which highlights the need to the detection of high risk population. Even more rare variants are likely to be involved in the pathogenesis, but due to the paucity of such variants, the detection of them is not straightforward. Candidate gene analysis may be worthwhile for the identification of predisposing genes and variants.

Hydrogen sulfide $\left(\mathrm{H}_{2} \mathrm{~S}\right)$ is a gas signaling molecule with various physiological activities in multiple organs (5). One of the major actions of $\mathrm{H}_{2} \mathrm{~S}$ is tissue protective activity in the gastrointestinal (GI) tract (6). Recently, D-amino oxidase (DAO) was found to be involved in a novel pathway for $\mathrm{H}_{2} \mathrm{~S}$ production by catalyzing D-cysteine $(7,8)$. D-cysteine is changed from L-cysteine by alkaline treatment and mostly provided from food (9). D-cysteine is easily absorbed through GI tract and enters the blood stream. Administration of D-cysteine has protective effect against ethanol-induced gastric mucosal damage through $\mathrm{DAO}-\mathrm{H}_{2} \mathrm{~S}$ signaling (10). To this end we hypothesized that DAO gene may be a candidate for susceptibility to gastric cancer.

\section{Materials and methods}

Study population. We employed the subjects registered in the Internet Database of Japanese single nucleotide polymorphisms for Geriatric Research (JG-SNP) (11). The study subjects comprised of consecutive autopsy cases collected at Tokyo Metropolitan Geriatric Hospital between 1995 and 2012. Autopsy procedures were performed on $\sim 29 \%$ of patients who died in the hospital. There were a total of 


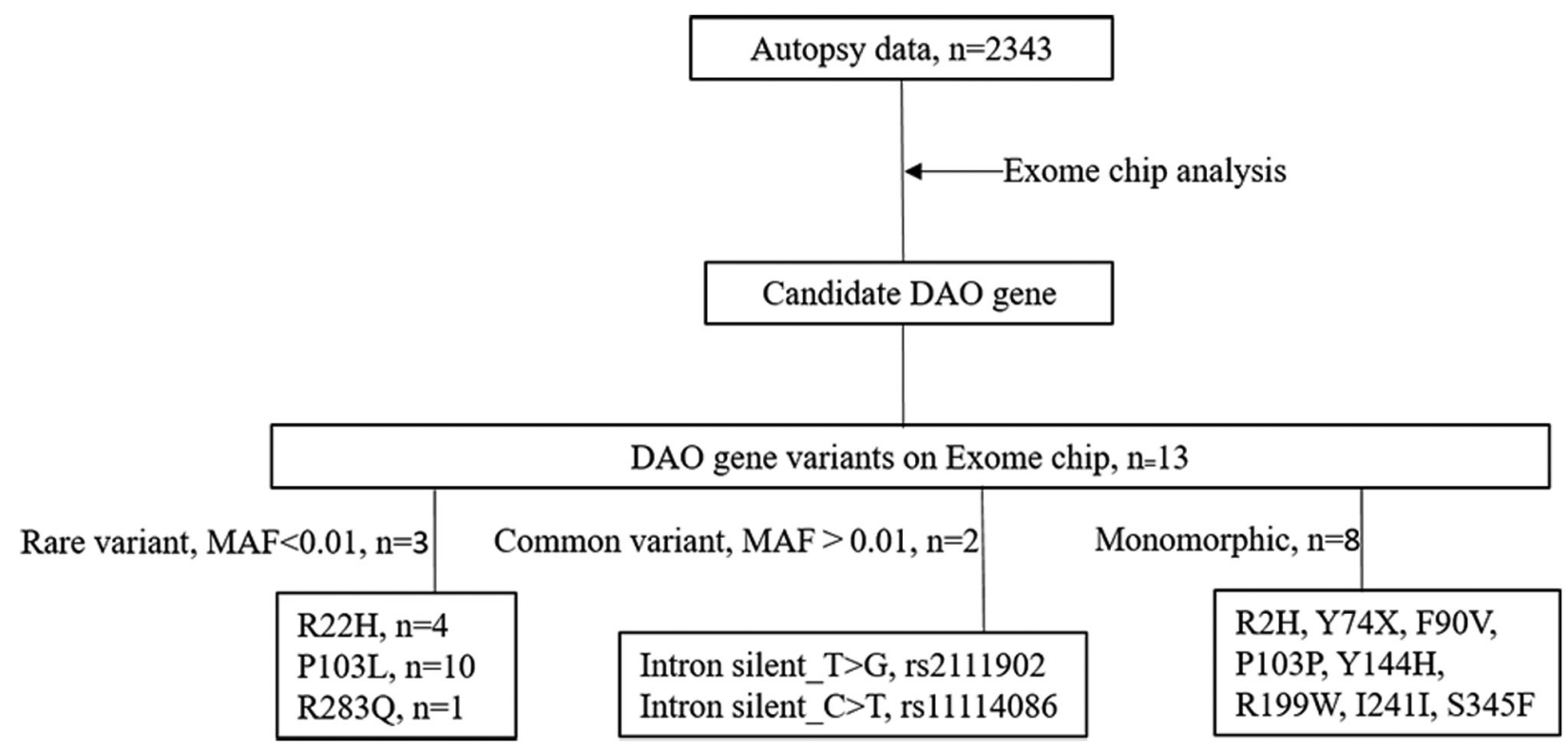

Figure 1. Flow chart of analysis. MAF, minor allele frequency; DAO, D-amino acid oxidase.

2,343 subjects, where 1,298 were men and 1,045 were women; the mean age at the time of death was 80 years. The presence or absence of any disease was determined by a thorough examination on autopsy. The detail of JG-SNP database can be seen elsewhere (11). Cancer-bearing subjects include those with any type of cancer, including occult cancer, found on autopsy. Smoking habit included both current smoking and ex-smoking. The distribution of any disease of the study group was not largely apart from those reported in a survey by the Ministry of Health, Labor, and Welfare of Japan (12).

Genotyping and statistical analysis. Genomic DNA was extracted from the renal cortex by a standard procedure using phenol and chloroform. All samples were genotyped with Illumina Infinium Human Exome Bead Chip Version 1.1 (Illumina, Inc.) by iScan in accordance with the Illumina protocols. Genotype calling was performed for all samples as a single project using the Genotyping Module (version 1.9) of the Genome Studio data analysis software package. Initial genotype clustering was performed using the default Illumina cluster file (Human Exome 12v1-1_A.egt) and manifest file (HumanExome-12v1-1_A.bpm) using the GenTrain 2 clustering algorithm. We considered a per sample call rate of $>98 \%$ as eligible, and 15 samples were excluded from the analysis. A total of 2,328 (99.4\%) out of the initial 2,343 subjects were successfully genotyped. Association of the rare variants with cancer state of the patients and P-value were completed via a Fisher's exact test using IBM SPSS Statistics software 25.0 (IBM Corp.).

The pathological assessment, genotyping and statistical analysis were performed in different institutions in a double-blind fashion to minimize bias.

Ethical statement. This study was approved by the Tokyo Medical and Dental University Ethics Committee (approval no. 2016-011-02), and the Tokyo Metropolitan Geriatric Hospital Ethics Committee (approval no. 230405).

\section{Results}

$D A O$ variants. On the exome chip, there were altogether 13 variants of the DAO gene. Among them, 8 variants were monomorphic, 2 common variants in the intron, and 3 rare $(\mathrm{MAF}<0.01)$ variants (Fig. 1).

Cancer bearing state of the heterozygotes of these three non-synonymous variants, p.R22H (rs200257378), p.P103L (rs200127576), and p.R283Q (rs143550642) were further analyzed (Table I). The number of p.R22H, p.P103L, and p.R283Q heterozygote carriers was four, ten and one, respectively. Thus, the minor allele frequency of these variants was 0.0009, 0.0021 and 0.0002, respectively. According to The Human Gene Mutation Database, the non-synonymous variants p.R22H, p.R283Q and p.P103L has been reported to be related with Frontotemporal dementia (13,14), Alzheimer's Disease (15) and amyotrophic lateral sclerosis (ALS), separately (16).

Characteristics of heterozygotes with DAO rare variants. Table II shows the demographic of the subjects carrying DAO rare variants. Among ten carriers of p.P103L, four were men and six were women. All of the men had gastric cancer. One man had an additional lung cancer. Among six women, one had colon cancer, one had small intestine cancer, one had multiple cancers of pancreas, breast and thyroid. Other three women had no cancer. All men had smoking and drinking habit. One woman had missing data, the others had no drinking habit and no smoking except for one smoker.

Four p.R22H carriers were all men, among them one man had malignant lymphoma and the others had no cancer. One p.R283Q carrier was a woman who had lung cancer with habit of smoking but not drinking.

Association of DAO p.P103L with cancer. Among 2,343 cases, $1,446(61.72 \%)$ were affected by different type of cancer. There are $262(11.18 \%)$ gastric cancer, $222(9.48 \%)$ colorectal cancer and $12(0.51 \%)$ small intestine cancer. Association of 
Table I. Cancer bearing state of the heterozygotes.

\begin{tabular}{|c|c|c|c|c|}
\hline SNV & Prediction $^{\mathrm{a}}$ & Heterozygotes & MAF & Related disease $^{\mathrm{b}}$ \\
\hline p.R22H & Benign & 4 & 0.0009 & Frontotemporal dementia \\
\hline p.P103L & Probably damaging & 10 & 0.0021 & Amyotrophic lateral sclerosis \\
\hline p.R283Q & Probably damaging & 1 & 0.0002 & Alzheimer's disease \\
\hline
\end{tabular}

aData obtained from PolyPhen2. ${ }^{b}$ Data obtained from Human Gene Mutation Database. MAF, minor allele frequency; SNV, single nucleotide variant.

Table II. Demographic and characteristics of heterozygotes with SNVs.

\begin{tabular}{|c|c|c|c|c|c|c|}
\hline Variant & Sample & Sex & Age & Cancer & Drinking & Smoking \\
\hline \multirow[t]{10}{*}{ p.P103L } & p.P103L 1 & M & 84 & Gastric & + & + \\
\hline & p.P103L 2 & M & 71 & Gastric & + & + \\
\hline & p.P103L 3 & M & 70 & Gastric & + & + \\
\hline & p.P103L 4 & M & 83 & Gastric, lung & + & + \\
\hline & p.P103L 5 & $\mathrm{~F}$ & 79 & Colon & - & - \\
\hline & p.P103L 6 & $\mathrm{~F}$ & 86 & Small Intestine & - & - \\
\hline & p.P103L 7 & $\mathrm{~F}$ & 96 & Pancreas, breast, thyroid & - & - \\
\hline & p.P103L 8 & $\mathrm{~F}$ & 93 & None & - & + \\
\hline & p.P103L 9 & $\mathrm{~F}$ & 93 & None & - & - \\
\hline & p.P103L 10 & $\mathrm{~F}$ & 82 & None & Missing & Missing \\
\hline \multirow[t]{4}{*}{ p.R22H } & p.R22H 1 & M & 69 & Malignant lymphoma & - & - \\
\hline & p.R22H 2 & M & 88 & None & Missing & Missing \\
\hline & p.R22H 3 & M & 91 & None & Missing & + \\
\hline & p.R22H 4 & M & 61 & None & Missing & - \\
\hline p.R283Q & p.R283Q & $\mathrm{F}$ & 71 & Lung & - & + \\
\hline
\end{tabular}

M, male; F, female; SNVs, single nucleotide variants.

Table III. p.P103L and cancer.

\begin{tabular}{|c|c|c|c|c|c|}
\hline Cancer & p.P103L (+) & p.P103L (-) & P-value & OR & $95 \% \mathrm{CI}$ \\
\hline $\mathrm{GC}(+)$ & 4 & 258 & 0.018 & 5.36 & $1.50-19.13$ \\
\hline GC (-) & 6 & 2075 & & & \\
\hline GIC (+) & 6 & 490 & 0.008 & 5.64 & $1.59-20.07$ \\
\hline GIC (-) & 4 & 1843 & & & \\
\hline
\end{tabular}

GC, gastric cancer; GIC, gastrointestinal cancer (gastric, colorectal and small intestine cancer). OR, odds ratio; CI, confidence interval.

gastric cancer and p.P103L variant showed positive result with $\mathrm{P}=0.018$ by Fisher's exact test, odds ratio, 5.36 and 95\% CI, 1.50-19.13 (Table III). Gastrointestinal cancer and p.P103L were also positively associated with $\mathrm{P}=0.008$, odds ratio, 5.64, and 95\% CI, 1.59-20.07 (Table III).

\section{Discussion}

We surveyed rare non-synonymous SNVs of the DAO gene in consecutive autopsy cases of the JG-SNP data base and found potential association between DAO p.P103L and gastric cancer in men. Due to the small sample size and the low allele frequency of the variant, this result should not be taken as a true association, but rather as a hypothesis generation, which needs to be confirmed in a larger size cohort study.

DAO is a flavin enzyme, which catalyzes oxidative deamination of natural D-amino acids. DAO gene is present in a single copy in human chromosome $12 \mathrm{q} 23-24$ region. It is ubiquitously expressed in tissues with high expression in liver, 
kidney and brain. Human DAO have various roles in modulating physical activity, including control of D-serine levels in the brain (17), production of $\mathrm{H}_{2} \mathrm{~S}$ from D-cysteine in brain, kidney and GI system $(6,8)$, and protection from microbes in the mucosa by generating $\mathrm{H}_{2} \mathrm{O}_{2}$ (9). Thus DAO appear to have important roles in the protection of GI tract by generating $\mathrm{H}_{2} \mathrm{~S}$ in the stomach and $\mathrm{H}_{2} \mathrm{O}_{2}$ in the intestine. This may in the same line that we found a sign of association between p.P103L and GI cancer (Table III).

Recent studies have shown detailed analysis of DAO non-synonymous variants and their structure function relationship (18). The p.P103L in our study has not been specified in the analysis, thus the functional consequence of this amino acid exchange is not fully understood. According to Polyphen2, a software to predict the impact of an amino acid substitution on the structure and function of a human protein, the p.R22H, p.P103L and p.R283Q are benign, probably damaging and probably damaging, respectively (Table I). Indeed, p. P103L has been reported as a phenotypic modulator of ALS (16). Nevertheless, none of the p.P103L carrier in our autopsy samples was diagnosed as ALS.

The striking difference in sex was seen for the effect of p.P103L on gastric cancer. While all of the heterozygote men were gastric cancer, none women heterozygote had gastric cancer but each one had colon cancer, intestine cancer and multiple cancers, respectively. According to the age-standardized incidence rate (ASIR) gastric cancer in men were generally twice than women (19). Although the reason for this gender difference is not clear, the fact that all four men were drinker and smoker might have accounted since these factors induce mucosal damage. While there were only three non-synonymous variants on the Exome Bead Chip, whole exome sequencing of familial ALS identified nine non-synonymous rare variants of p.P103L, p.E121K, p.R199Q, p.R199W, p.Q201R, p.T269I, p.A323V, p.G331E and p.S345F, according to the Human Genome Mutation Database (HGMD). It would be interesting to study whether other hypomorphic DAO variants also associate with gastric cancer, since co-occurrence of ALS and gastric cancer in Japanese are reported (20).

In conclusion, we found a cluster of DAO rare variant p.P103L carriers in gastric cancer men. DAO variants warrants further study with regard to GI diseases in larger size cohort.

\section{Acknowledgements}

Not applicable.

\section{Funding}

The present study was funded by the Ministry of Education, Culture, Sports, Science and Technology/Japan Society for the Promotion of Science (grant no. 17 K09081).

\section{Availability of data and materials}

The datasets used and/or analyzed during the present study are available from the corresponding author on reasonable request.

\section{Authors' contributions}

MM designed the present study. TA performed pathological analysis. MT acquired and analyzed clinical data. YZ performed genotyping and statistical analyses. MM, TA and MT confirm the authenticity of all the raw data. YZ and MM wrote the manuscript. All authors read and approved the manuscript.

\section{Ethics approval and consent to participate}

The study protocol was approved by the Ethics Committees of Tokyo Geriatric Hospital and Tokyo Medical and Dental University and authorized by TMDU Research Ethics Committee (approval no. 2016-011-02). Written informed consent was obtained from family member of all participants involved in the present study before autopsy.

\section{Patient consent for publication}

Not applicable.

\section{Competing interests}

The authors declare that they have no competing interests.

\section{References}

1. Lott PC and Carvajal-Carmona LG: Resolving gastric cancer aetiology: An update in genetic predisposition. Lancet Gastroenterol Hepatol 3: 874-883, 2018.

2. Rahman R, Asombang AW and Ibdah JA: Characteristics of gastric cancer in Asia. World J Gastroenterol 20: 4483-4490, 2014.

3. Yusefi AR, Bagheri Lankarani K, Bastani P, Radinmanesh M and Kavosi Z: Risk factors for gastric cancer: A systematic review. Asian Pac J Cancer Prev 19: 591-603, 2018.

4. Huang KL, Mashl RJ, Wu Y, Ritter DI, Wang J, Oh C, Paczkowska M, Reynolds S, Wyczalkowski MA, Oak N, et al: Pathogenic germline variants in 10,389 adult cancers. Cell 173: 355-370.e14, 2018.

5. Kimura H: Signalling by hydrogen sulfide and polysulfides via protein S-sulfuration. Br J Pharmacol 177: 720-733, 2020.

6. Shen F, Zhao CS, Shen MF, Wang Z and Chen G: The role of hydrogen sulfide in gastric mucosal damage. Med Gas Res 9: 88-92, 2019.

7. Shibuya N, Koike S, Tanaka M, Ishigami-Yuasa M, Kimura Y, Ogasawara Y, Fukui K, Nagahara N and Kimura H: A novel pathway for the production of hydrogen sulfide from D-cysteine in mammalian cells. Nat Commun 4: 1366, 2013.

8. Tang S, Huang D, An N, Chen D and Zhao D: A novel pathway for the production of $\mathrm{H} 2 \mathrm{~S}$ by DAO in rat jejunum. Neurogastroenterol Motil 28: 687-692, 2016.

9. Sasabe J, Miyoshi Y, Rakoff-Nahoum S, Zhang T, Mita M, Davis BM, Hamase K and Waldor MK: Interplay between microbial d-amino acids and host d-amino acid oxidase modifies murine mucosal defence and gut microbiota. Nat Microbiol 1: 16125, 2016.

10. Souza LK, Araújo TS, Sousa NA, Sousa FB, Nogueira KM, Nicolau LA and Medeiros JV: Evidence that d-cysteine protects mice from gastric damage via hydrogen sulfide produced by d-amino acid oxidase. Nitric Oxide 64: 1-6, 2017.

11. Sawabe M, Arai T, Kasahara I, Esaki Y, Nakahara K, Hosoi T, Orimo H, Takubo K, Murayama S and Tanaka N: Developments of geriatric autopsy database and Internet-based database of Japanese single nucleotide polymorphisms for geriatric research (JG-SNP). Mech Ageing Dev 125: 547-552, 2004.

12. Oda K, Tanaka N, Arai T, Araki J, Song Y, Zhang L, Kuchiba A, Hosoi T, Shirasawa T, Muramatsu $M$ and Sawabe M: Polymorphisms in pro- and anti-inflammatory cytokine genes and susceptibility to atherosclerosis: A pathological study of 1503 consecutive autopsy cases. Hum Mol Genet 16: 592-599, 2007. 
13. Kim EJ, Kim YE, Jang JH, Cho EH, Na DL, Seo SW, Jung NY, Jeong JH, Kwon JC, Park KH, et al: Analysis of frontotemporal dementia, amyotrophic lateral sclerosis, and other dementia-related genes in 107 Korean patients with frontotemporal dementia. Neurobiol Aging 72: 186.e1-186.e7, 2018.

14. Zhang H, Cai W, Chen S, Liang J, Wang Z, Ren Y, Liu W, Zhang X, Sun Z and Huang X: Screening for possible oligogenic pathogenesis in Chinese sporadic ALS patients. Amyotroph Lateral Scler Frontotemporal Degener 19: 419-425, 2018.

15. Barber IS, Braae A, Clement N, Patel T, Guetta-Baranes T, Brookes K, Medway C, Chappell S, Guerreiro R, Bras J, et al: Mutation analysis of sporadic early-onset Alzheimer's disease using the NeuroX array. Neurobiol Aging 49: 215.e1-215.e8, 2017.

16. Pang SY, Hsu JS, Teo KC, Li Y, Kung MHW, Cheah KSE, Chan D, Cheung KMC, Li M, Sham PC and Ho SL: Burden of rare variants in ALS genes influences survival in familial and sporadic ALS. Neurobiol Aging 58: 238.e9-238.e15, 2017.

17. Pollegioni L, Sacchi S and Murtas G: Human D-Amino acid oxidase: Structure, function, and regulation. Front Mol Biosci 5: 107, 2018.
18. Sacchi S, Cappelletti P and Murtas G: Biochemical properties of human D-amino acid oxidase variants and their potential significance in pathologies. Front Mol Biosci 5: 55, 2018.

19. Ang TL and Fock KM: Clinical epidemiology of gastric cancer. Singapore Med J 55: 621-628, 2014.

20. Nozaki I, Kato-Motozaki Y, Ikeda T, Tagami A, Takahashi K, Ishida $\mathrm{C}$ and Komai $\mathrm{K}$ : Clinical features in association with neurodegenerative diseases and malignancies. Eur Neurol 71: 99-105, 2014

This work is licensed under a Creative Commons Attribution-NonCommercial-NoDerivatives 4.0 International (CC BY-NC-ND 4.0) License. 